\title{
Recomendação Personalizada de Conteúdo Instrucional Complementar usando Repositório de Objetos de Aprendizagem e Recursos da Web
}

\author{
Cleon Xavier Pereira Junior ${ }^{1,2}$, Rafael Dias Araújo ${ }^{1}$, Fabiano Azevedo Dorça ${ }^{1}$ \\ ${ }^{1}$ Universidade Federal de Uberlândia (UFU) \\ Uberlândia, MG - Brasil \\ ${ }^{2}$ Instituto Federal Goiano (IF Goiano) \\ Iporá, GO - Brasil \\ cleon.junior@ifgoiano.edu.br, \{rafael.araujo, fabianodor\}@ufu.br
}

\begin{abstract}
This article presents a proposal for personalized recommendation of Learning Objects (LOs) in the form of complementary content for a class. Considering that educational repositories are more restricted than existing content on the Web, this work also investigates the extension of materials, using Wikipedia and Youtube to better support the student's learning process. The proposal uses Content-Based Filtering (CBF), taking into account students' preferences and classes metadata. The results show that the support of external content generates better adaptability and diversification in the instructional content delivered to the student.
\end{abstract}

Resumo. Este artigo apresenta uma proposta de recomendação personalizada de Objetos de Aprendizagem (OA) na forma de conteúdo complementar (ou material adicional) a uma aula. Considerando que os repositórios educacionais são mais restritos que o conteúdo existente na Web, este trabalho também investiga a extensão de materiais, utilizando Wikipedia e Youtube para oferecer melhor suporte ao processo de aprendizagem do estudante. A proposta utiliza Filtragem Baseada em Conteúdo (FBC), levando em consideração preferências do estudante e metadados de uma aula. Os resultados apontam que o suporte de conteúdos externos gera melhor adaptatividade e diversificação no conteúdo instrucional entregue ao estudante.

\section{Introdução}

Sistemas de Recomendação (SR) que oferecem recursos de maneira personalizada aos usuários têm sido explorados nas diversas aplicações computacionais [Lops et al. 2011]. Com o crescente número de conteúdos disponíveis, os serviços personalizados são encontrados com mais frequência. Esse tipo de serviço já está associado no cotidiano dos usuários, através, principalmente, do comércio eletrônico [Pappas et al. 2017]. Na área da educação, os esforços em pesquisas que envolvem recomendação personalizada em ambientes de aprendizagem também vêm ocorrendo [Manouselis et al. 2011].

Uma das estratégias utilizadas pelos SR para prover recursos ao usuário é conhecida por Filtragem Baseada em Conteúdo (FBC) [Lops et al. 2011]. No contexto educacional, quando aplicada a FBC para recomendação de Objetos de Aprendizagem (OA), 
espera-se encontrar os recursos que mais se aproximam do perfil do estudante que foi selecionado para receber a recomendação. Ocasionalmente, essas abordagens se limitam a explorar somente os repositórios onde são integradas, que, por vezes, possuem metadados de OA estruturados em algum padrão. Tal restrição garante uma qualidade e controle do conteúdo e facilita no processo de recomendação, porém limita a possibilidade de variação e enriquecimento na adaptação para diferentes perfis.

Enquanto que os repositórios de OA possuem uma limitação na quantidade de materiais, a Web possui um acervo imensurável de conteúdos não-estruturados. Com rápidas buscas, é possível encontrar vídeos, imagens, textos, áudios e outros formatos de mídia relacionados a um determinado conceito. Alguns materiais desse acervo, acessado frequentemente por páginas da Web, não foram necessariamente desenvolvidos para $o$ processo de aprendizagem. Além disso, as máquinas de busca, como o Google, que trazem esses materiais, estão mais preocupadas com a relevância do domínio do conteúdo, número de palavras-chave correspondentes, segurança, frequência de acessos, dentre outros $^{1}$. Fatores ligados à personalidade do usuário e conhecimentos prévios, por exemplo, não são levados em consideração.

Diante da quantidade de recursos disponíveis atualmente em páginas da Web, exige-se das abordagens de recomendação de conteúdos uma preocupação que vai além de busca em repositórios de OA [De Medio et al. 2019]. Para os SR educacionais, os desafios que surgem ao explorar páginas da Web envolvem a questão da qualidade do recurso e do processo de recomendação, uma vez que as técnicas existentes estão adaptadas para a exploração de metadados de OA. Um caminho para a exploração dos recursos mencionados é a estruturação destes em algum padrão de metadados de OA.

Algumas plataformas, como a Wikipedia ${ }^{2}$ e o Youtube $^{3}$, além de estruturarem seus conteúdos em páginas Web, também fornecem API (Application Programming Interface) que permitem extrair os metadados existentes nas páginas, como título, descrição, avaliações de usuários, dentre outros. Tais metadados podem auxiliar na estruturação destes recursos como OA [Júnior and Dorça 2018, Pal et al. 2019], o que permite, a partir daí, o uso de técnicas já existentes para o processo de recomendação.

Pesquisas apontam que materiais adicionais durante a apresentação de uma aula podem trazer benefícios para a aprendizagem [Ljubojevic et al. 2014]. Além disso, a adaptatividade no processo de aprendizagem pode gerar benefícios para o estudante. Neste aspecto, este trabalho apresenta uma abordagem de recomendação personalizada de materiais adicionais, baseada em FBC, que combina OA de um repositório educacional e páginas da Web que dispõem de API.

\section{Fundamentação Teórica e Trabalhos Correlatos}

É possível encontrar três maneiras distintas de filtragens de recursos de aprendizagem em um SR, sendo elas: Filtragem Colaborativa (FC); Filtragem Baseada em Conteúdo (FBC) e Filtragem Híbrida (FH) [Wan and Niu 2018]. Um exemplo do uso da FC se dá através da geração de uma matriz de avaliações de usuários para um conjunto de produtos (sendo

\footnotetext{
${ }^{1}$ https://backlinko.com/google-ranking-factors

${ }^{2}$ https://www.wikipedia.org/

${ }^{3}$ https://www.youtube.com/
} 
que os usuários e produtos representam as linhas e colunas) e posterior recomendação a partir desse conjunto de dados [Almahairi et al. 2015]. O problema desse modelo para um SR é a necessidade da classificação de usuários com preferências similares para conseguir fazer uma recomendação para o sujeito interessado [Lops et al. 2011]. Existem melhorias para esse tipo de abordagem, conforme pode ser observado em [Almahairi et al. 2015].

Os SR que possuem FBC buscam combinar o perfil do usuário com atributos do conteúdo para recomendar novos itens para os interessados. A vantagem de usar esse tipo de aplicação é que, para um determinado usuário, a recomendação vai ocorrer a partir das características dele e dos recursos disponíveis. Ou seja, nesse tipo de abordagem não há uma dependência do conjunto de usuários para realizar a recomendação [Lops et al. 2011]. Abordagens que utilizam FBC possuem limitações no número e tipo de características que serão utilizadas como associação para a recomendação de objetos.

FBC são comumente encontradas em ambientes de recomendação de Objetos de Aprendizagem (OA). O trabalho de [Wan and Niu 2018] apresenta um SR de OA baseado em auto-organização. O SR proposto utiliza FBC para recomendação personalizada e faz uma crítica à necessidade de levar em consideração as mudanças dos estudantes no processo de aprendizagem. Tal fator é comumente ignorado quando se trata de FBC, pois, uma vez capturada as características dos estudantes que serão levadas em consideração para o processo de recomendação, estas se tornam estáticas e não são atualizadas no decorrer do processo.

Outra crítica à FBC apontada por [Wan and Niu 2018] é com relação a baixa diversidade, indicando sempre recomendações extremamente similares por consequência da técnica utilizada, uma vez que a abordagem visa encontrar os OA que mais se assemelham ao perfil do estudante. Com relação à baixa diversidade, além da técnica utilizada, outro motivo que pode ocasionar são as limitações de OA em repositórios. Além de, por vezes, não cobrir todos os conceitos que um estudante necessita, há uma necessidade em explorar páginas da Web como recursos educacionais, permitindo assim ganho nas possibilidades de recomendação de OA [De Medio et al. 2019].

O trabalho desenvolvido por [Ljubojevic et al. 2014] apresenta as vantagens do uso de vídeos educacionais como materiais adicionais para uma aula. Além disso, a pesquisa conclui que o melhor lugar para estes vídeos aparecerem é no meio de uma aula. Ainda no aspecto de conteúdo complementar no processo de aprendizagem, o trabalho de [Selwyn and Gorard 2016] fez um levantamento com 1658 estudantes australianos acerca do uso da Wikipedia. A pesquisa aponta que a maioria dos estudantes têm utilizado Wikipedia como fonte de pesquisa para estudos introdutórios e/ou adicionais. Além disso, o Youtube aparece também como uso.

Diferente da Wikipedia em relação ao formato das mídias, porém ainda estruturado no formato de páginas da Web, o Youtube também tem sido bastante explorando para o contexto de aprendizagem. A aprendizagem baseada em vídeo possui vantagens por proporcionar um estudo mais individualizado e flexível [Pal et al. 2019]. O trabalho de [Curran et al. 2020] apresenta uma revisão da literatura sobre o uso do Youtube como recurso educacional na área de medicina. 


\section{Abordagem Proposta}

Este trabalho apresenta a proposta de uma abordagem para recomendação personalizada de materiais adicionais, a partir do conteúdo de uma aula e preferências do estudante. $\mathrm{O}$ SR conta com FBC e é capaz de recomendar OA presentes no repositório local e também em páginas da Web. A Figura 1 é uma visão geral da abordagem.

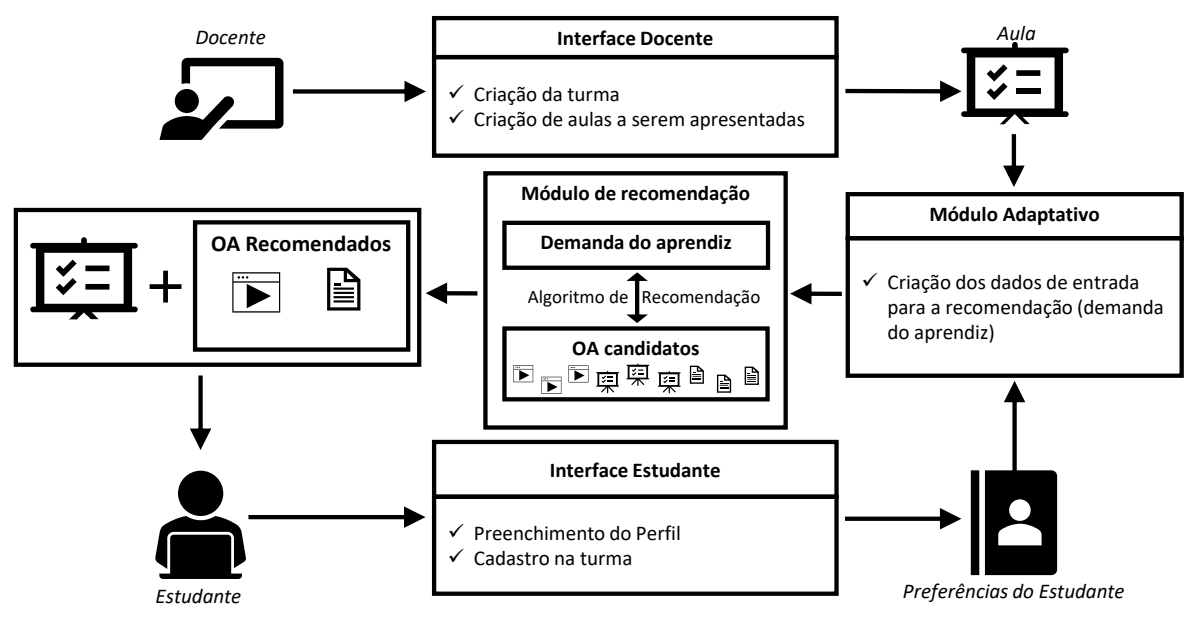

Figura 1. Visão geral do SR baseado em FBC.

Nota-se na Figura 1 que, inicialmente, é esperado que docentes criem turmas e cadastrem aulas para essas turmas. Para cada aula, os docentes devem inserir, obrigatoriamente: (i) Título; (ii) Palavras-chave acerca do conteúdo; e, (iii) arquivo contendo a apresentação da aula. Além dos itens obrigatórios, também é possível cadastrar outros metadados baseados no padrão IEEE-LOM [IEEE LTSC 2001]. Ao término do preenchimento, o material e seus metadados são armazenados no repositório como um OA.

Relacionado aos estudantes, esses preenchem os dados pessoais (nome, data de nascimento, turma, dentre outros) e questionários que irão traçar as preferências e serão utilizados para o processo de recomendação. Atualmente, para efeitos experimentais, esta abordagem considera um modelo probabilístico de EA do estudante baseado na teoria de Felder e Silverman [Araújo et al. 2020, Felder et al. 1988]. A Tabela 1 apresenta um exemplo considerando este modelo, que não classifica o estudante de maneira categórica e rígida, mas armazena uma probabilidade de preferência por um ou outro estilo dentro de cada dimensão como forma de apoiar a recomendação personalizada de conteúdo.

Tabela 1. Exemplo de classificação probabilística do EA de estudante.

\begin{tabular}{cccccccc}
\hline \multicolumn{2}{c}{ Entrada } & \multicolumn{2}{c}{ Organização } & \multicolumn{2}{c}{ Processamento } & \multicolumn{2}{c}{ Percepção } \\
\hline Visual & Verbal & Global & Sequencial & Ativo & Reflexivo & Sensitivo & Intuitivo \\
0.9095 & 0.0905 & 0.1815 & 0.8185 & 0.0995 & 0.9005 & 1.0000 & 0.0000 \\
\hline
\end{tabular}

É importante pontuar que existem trabalhos que questionam a efetividade do uso de EA no processo educacional [An and Carr 2017, Kirschner 2017]. No entanto, conforme observado por [Araújo et al. 2020], as críticas dizem respeito à classificação feita exclusivamente com EA e, também, sem considerar que os EA podem ser alterados no decorrer do processo. Então, neste trabalho, EA são considerados uma tendência, e não uma certeza categórica, em relação às preferências do estudante. Além disso, esta abor- 
dagem utiliza os EA combinados com tópicos abordados em uma aula, e dinamicamente atualiza os EA ao longo do tempo.

A partir dos metadados da aula, previamente cadastrados pelo docente, e das preferências do estudante, o Módulo Adaptativo fica responsável por gerar a demanda do estudante, que são os dados de entrada para o Módulo de Recomendação conseguir filtrar os melhores OA. Uma ontologia foi modelada para que, através de regras SWRL (Semantic Web Rule Languages), fosse possível inferir os metadados esperados de um OA. As regras foram criadas através das relações entre EA e campos do IEEE-LOM [Araújo 2017]. Considerando um estudante fictício (apresentado na Tabela 1) matriculado em uma turma de Introdução à Programação, a Tabela 2 apresenta a demanda desse estudante gerada a partir das regras de inferência.

Tabela 2. Exemplo de entrada de dados para o processo de recomendação.

\begin{tabular}{|l|lll|}
\hline Título & Estrutura de Seleção & & \\
\hline Palavras-chave & Linguagem C & if & \\
\hline Tipo de Recurso & Lecture & Self Assessment & Figure \\
Educacional & Summary & Graph & Definition \\
& Narrative Text & Table & Scenario \\
& Illustration & Glossary & Index \\
& Diagram & Presentation & Guideline \\
& Example & Practice & Experiment \\
& Simulation & Demonstration & Attractor \\
& Additional Resource & Nonexample & Guidance \\
& Slide & Note & Analogy \\
\hline Tipo de Interatividade & Expositive & Mixed & \\
\hline Nível de interatividade & Very Low & Low & Medium \\
\hline Formato & Image & Application & Video \\
\hline
\end{tabular}

Com o objetivo de conseguir associar conteúdo da Web com dados apresentados na Tabela 2, foram escolhidos inicialmente a Wikipedia e o Youtube como bases de busca externas, pelo fato de fornecerem API que permitem extrair metadados das páginas, possibilitando associá-los com metadados do padrão IEEE-LOM. A Tabela 3 apresenta um mapeamento da estruturação das páginas do Youtube e Wikipedia em metadados de OA. A partir do padrão IEEE-LOM, levando em consideração os metadados que a API fornece e as limitações das bases (como ausência de interatividade, por exemplo), é possível gerar os principais metadados para permitir o uso da FBC na abordagem.

Tabela 3. Estruturação do Youtube e Wikipedia no padrão IEEE-LOM.

\begin{tabular}{l|l|l}
\hline Metadados de OA & Wikipedia & Youtube \\
\hline Título & Título da página & Titulo do vídeo \\
Descrição & Resumo da página & Descrição do vídeo \\
Palavras-chave & Conceitos utilizados na busca & Conceitos utilizados na busca \\
Idioma & Idioma da Página & Idioma do vídeo \\
Tipo de interatividade & Expositivo & Expositivo \\
Nível de interatividade & Baixo & Baixo \\
Recursos de aprendizagem & Introdução; definição; visão geral; & Apresentação; demonstração; analogia; \\
Formato & resumo; figura; referência & extração pela narração ou descrição \\
\hline
\end{tabular}

Além da extração de metadados, as API da Wikipedia e do Youtube dispõem de máquinas de busca para procurar os recursos através de cadeias de cadeias de caracteres. 
Neste trabalho, as buscas ocorrem por meio da combinação das palavras-chave com o título da aula. Após experimentações acerca da qualidade e relação dos resultados retornados com o conteúdo desejado, notou-se que seria possível utilizar as máquinas de busca limitando aos primeiros resultados. Portanto, para cada palavra-chave, são selecionados até cinco vídeos do Youtube (utilizando o filtro para buscar somente da categoria educacional) e até três páginas da Wikipedia. Se uma busca retorna o mesmo resultado para duas ou mais palavras-chave distintas, considera-se que este recurso dá suporte para todas as palavras-chave que eles são retornados.

Com relação aos recursos de aprendizagem, o uso de API é mais uma vez necessário. Para a Wikipedia, automaticamente são anotados os recursos de introdução, definição, visão geral e resumo. Através da API é possível verificar se uma página tem figura e/ou referência. Portanto, para cada página também é verificado a existência de figura e/ou referência e anotado, caso contenha. Já o Youtube é anotado apresentação, demonstração e analogia de forma automática. Os outros recursos de aprendizagem são extraídos da narração do vídeo a partir de técnicas de processamento de linguagem natural, realizando as etapas de tokenize, remoção de palavras vazias (stop words) e stemming.

Ao finalizar a anotação dos metadados de OA nos recursos da Web, a abordagem avança para o módulo de recomendação. Nessa etapa, o objetivo é encontrar um ou mais OA que estejam próximos às demandas do estudante. Espera-se que os OA recomendados cubram todas os termos do parâmetro palavras-chave da demanda do estudante. Nesse sentido, esta abordagem utiliza um Algoritmo Genético (AG) conforme apresentado inicialmente por [Júnior and Dorça 2018] e adaptado ao contexto deste trabalho, onde o AG é criado para resolver o problema de cobertura por conjuntos. Sendo assim, cada OA é comparado com a demanda do estudante e cada conjunto de OA que consegue cobrir todas as palavras-chave pode ser uma solução para o problema. O desafio é encontrar a melhor solução, isto é, a melhor combinação de OA que consiga cobrir todas as palavraschave. Antes de dar prosseguimento ao processo de recomendação, é necessário entender como é calculado o custo de cada OA. A função $c_{j}$ representa o custo do OA potencial a ser recomendado (isto é, que cobre ao menos uma das palavras-chave):

$$
c_{\mathrm{j}}=\sum_{i=1}^{y} \theta_{\mathrm{i}}
$$

onde: $\theta i$ representa o resultado da comparação $(\theta i=[0,1])$ entre o parâmetro da demanda do estudante e do OA; e y representa os parâmetros utilizados para realizar o cálculo do $c_{j}$. Para esta abordagem, os itens da Tabela 2, com exceção do título, são utilizados como parâmetros. Quanto mais próximo de zero for o $c_{j}$, mais semelhante é este OA com as preferências do estudante. Um cromossomo do AG é a representação de uma solução viável, podendo ter um ou mais OA. A soma $\operatorname{dos} c_{j}$ dos OA representa o fitness.

Para os parâmetros de tipos de recursos de aprendizagem e palavras-chave são aplicados um cálculo da similaridade de cossenos. Para cada parâmetro, duas cadeias de caracteres são criadas (demanda e OA candidato) e comparadas. Quanto mais similares, mais próximo de 1 é o resultado. Portanto, realiza-se a subtração de 1 pelo resultado da similaridade para obter o resultado inversamente proporcional. Para os demais parâmetros, cada item do vocabulário do campo recebe um valor numérico. Caso o valor do OA seja igual ao representado pela demanda, o cálculo de $\theta i$ inicialmente resultará em zero. Se 
forem distintos, o cálculo inicial resultará em um número maior que zero, podendo chegar a 1 caso sejam opostos (por exemplo, o OA ter o nível de interatividade 'muito baixo' e a demanda do estudante o nível 'muito alto'). Após os cálculos iniciais, os $\theta i$ são atualizados levando em consideração os valores probabilísticos de cada dimensão do EA, conforme apresentado na Tabela 1.

Depois de realizar os cálculos dos custos dos OA candidatos (oriundos do repositório local da aplicação, Wikipedia e Youtube) e criar a população inicial com combinações de OA, o AG é executado e retorna uma solução. O estudante então recebe os materiais adicionais enquanto acessa a apresentação de uma aula. A Figura 2 traz uma solução para a demanda apresentada na Tabela 2.

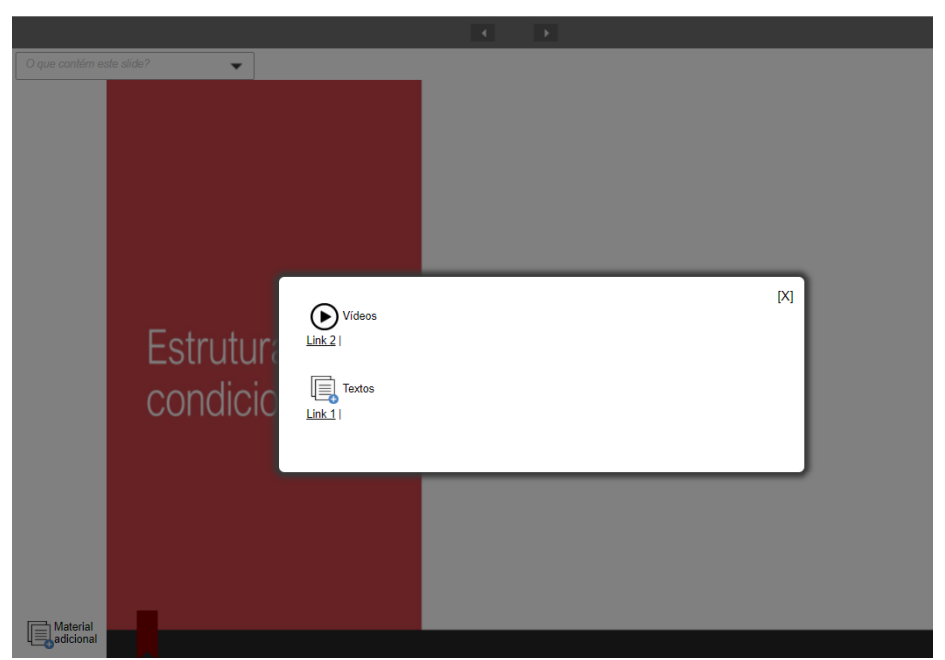

Figura 2. Exemplo de recomendação de materiais adicionais a partir de uma aula.

A Figura 3(a) apresenta como ocorre a renderização de um vídeo para o estudante e a Figura 3(b) apresenta a renderização de uma página da Wikipedia. Além disso, como já mencionado, é possível que a recomendação traga OA do repositório local em que a abordagem está inserida. É interessante que os SR consigam realizar uma diversificação nos resultados, tentando aproximar do interesse do usuário. Portanto, na próxima seção são apresentados testes para verificar a diversidade de materiais retornados e as recomendações distintas encontradas.

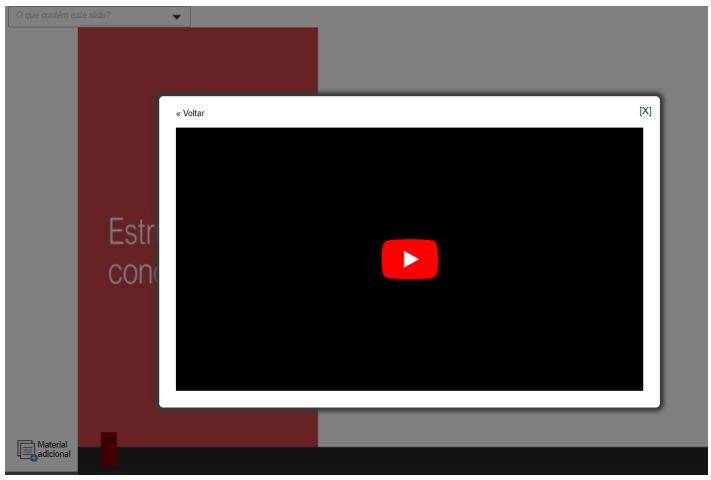

(a) Conteúdo complementar oriundo do Youtube

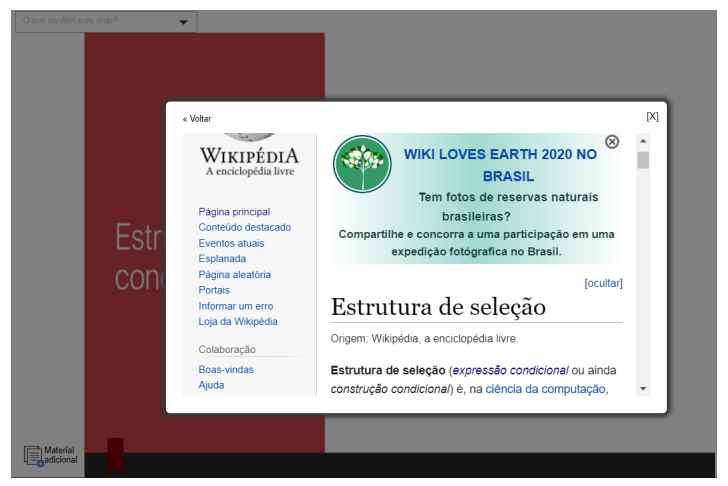

(b) Conteúdo complementar oriundo da Wikipedia

Figura 3. Exemplo de recomendação de materiais do Youtube e da Wikipedia. 


\section{Experimentos e Resultados}

A fim de verificar a capacidade da abordagem em gerar recomendação personalizada e apresentar uma diversificação na entrega de materiais adicionais, foi feita uma análise a partir de um banco de dados com EA probabilísticos de estudantes coletados por meio da plataforma Classroom eXperience (CX). A primeira etapa consistiu no levantamento do perfil de 61 estudantes que possuíam classificação probabilística dos EA. A Figura 4 apresenta a distribuição dos estudantes (em porcentagem) de acordo com os EA.

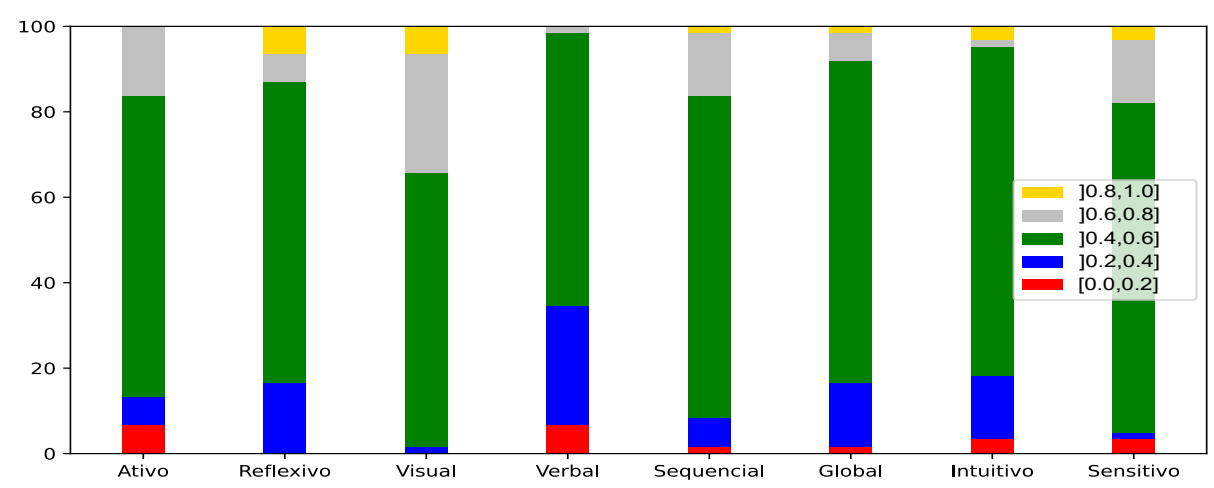

Figura 4. Distribuição dos estudantes por EA na base analisada.

Ao analisar a figura, é possível verificar que a maioria dos estudantes estão classificados com valores entre 0.4 e 0.6 (perfis entre 40 e $60 \%$ para cada EA). Isso reforça a necessidade de uma recomendação utilizando classificação probabilística, onde a distância do OA com a demanda do estudante é calculada levando em consideração o valor probabilístico do EA do estudante, ao contrário de uma classificação binária/excludente, categórica e estática. Se o processo fosse realizado utilizando classificações binárias e estáticas para cada entrada, colocariam os EA dos alunos em extremos e forçaria uma recomendação baseada nesses extremos. Por exemplo, um aluno com valor probabilístico de 50,01\% para o estilo visual (dimensão Entrada) seria classificado exclusivamente como visual, ao passo que ele está mais centralizado e com preferências tão próximas do visual quanto do verbal.

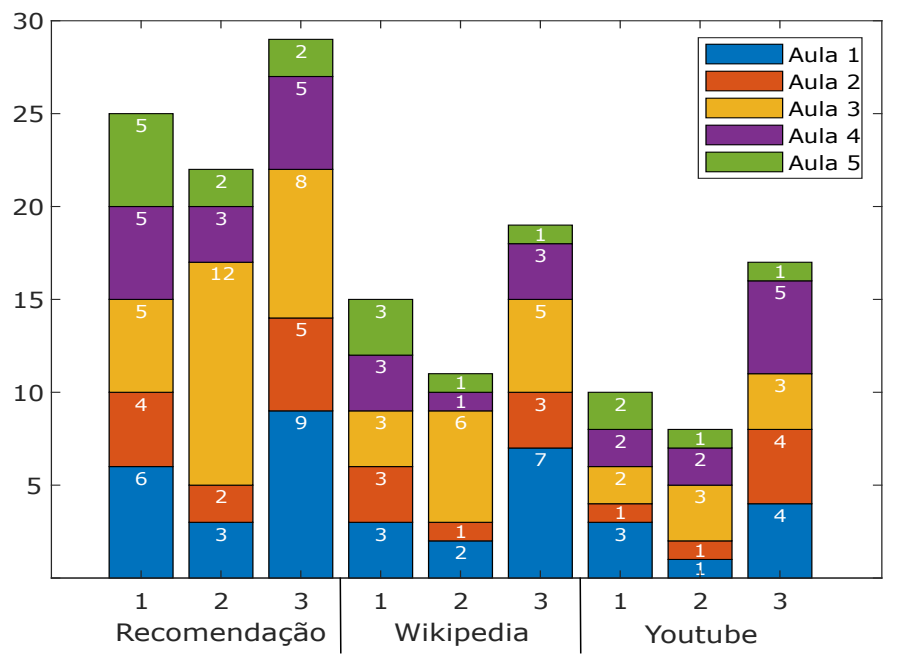

Figura 5. Quantidade de recomendações distintas e OA por aula. 
Com relação aos metadados de aula, necessários para realizar a recomendação, foram criadas cinco aulas com assuntos do domínio de computação para realização dos testes. Para cada aula, foram realizados testes com até três palavras-chave e, considerando como candidatos, os OA oriundos da Wikipedia, do Youtube e do repositório local em que a abordagem foi inserida. A Figura 5 apresenta o número de recomendações distintas encontradas de acordo com a quantidade de palavras-chave informadas. OA oriundos do repositório local apareceram como candidatos mas não foram selecionados por não apresentarem proximidade com a demanda do estudante.

A partir dos dados apresentados na Figura 5 é possível notar que, para uma palavra-chave, em geral, a quantidade de recomendações distintas realizadas, levando em consideração os 61 perfis, foi maior que para duas. Isso ocorre, pois, quando se tem somente uma palavra-chave, um único OA consegue cobri-la e vários OA candidatos possuem o mesmo custo. A partir de duas palavras-chave, a solução apresentada consegue realizar recomendações mais direcionadas para o conteúdo da aula. Com três palavras-chave, além de aumentar o número de recomendação distintas, observou-se que foi preciso no mínimo dois OA para cobri-las, em todas as cinco aulas. Ainda observando o gráfico da Figura 5, nota-se que a Aula 2 apresenta um comportamento diferente, trazendo um número maior de recomendações com duas palavras-chave se comparada com uma única. Ao observar os OA retornados, foi possível verificar que a maioria era da Wikipedia e que não estava tão relacionado ao assunto, como é esperado pela abordagem. Apesar da busca da Wikipedia selecionar somente os três melhores resultados para cada palavra-chave, ainda é um número alto e corre um risco de se recomendar algo que não seja tão útil para acréscimo de conhecimento ao estudante. Nota-se que, para a Wikipedia, no momento de buscar OA candidatos pela API, o ideal é que seja selecionado somente o melhor resultado por palavra-chave.

\section{Considerações finais e trabalhos futuros}

Este trabalho apresentou uma proposta de recomendação personalizada utilizando FBC para fornecer materiais adicionais ao estudante, de acordo com as preferências, durante o acesso a uma aula em um ambiente de aprendizagem. O trabalho, além de realizar buscas no repositório local do ambiente de aprendizagem, também explorou a Wikipedia e o Youtube. De acordo com os critérios utilizados para a recomendação, resultados apontaram que os materiais da Wikipedia e do Youtube tiveram maior sucesso na recomendação e foi notado que, para este tipo de abordagem, é interessante que se tenha mais de uma palavra-chave relacionada ao conteúdo da aula. Esta abordagem está sendo integrada ao ambiente CX, descrito em [Araújo 2017], com intuito de melhorar a experiência e o processo de aprendizagem dos estudantes. Conforme experimentos preliminares apresentados, a abordagem entrega de forma satisfatória conteúdos complementares a partir da Web. Experimentos e análises estatísticas com estudantes serão realizados com intuito de se verificar o quanto esta abordagem contribui para melhorar a performance dos estudantes nos cursos.

\section{Referências}

Almahairi, A., Kastner, K., Cho, K., and Courville, A. (2015). Learning distributed representations from reviews for collaborative filtering. In Proceedings of the 9th ACM Conference on Recommender Systems, pages 147-154. 
IX Congresso Brasileiro de Informática na Educação (CBIE 2020)

Anais do XXXI Simpósio Brasileiro de Informática na Educação (SBIE 2020)

An, D. and Carr, M. (2017). Learning styles theory fails to explain learning and achievement: Recommendations for alternative approaches. Personality and Individual Differences, 116:410-416.

Araújo, R. D. (2017). Uma Arquitetura Computacional para Autoria e Personalização de Objetos de Aprendizagem em Ambientes Educacionais Ubíquos. PhD thesis, Universidade Federal de Uberlândia.

Araújo, R. D., Brant-Ribeiro, T., Ferreira, H. N., Dorça, F. A., and Cattelan, R. G. (2020). Using learning styles for creating and personalizing educational content in ubiquitous learning environments. Revista Brasileira de Informática na Educação, 28:133.

Curran, V., Simmons, K., Matthews, L., Fleet, L., Gustafson, D. L., Fairbridge, N. A., and $\mathrm{Xu}, \mathrm{X}$. (2020). Youtube as an educational resource in medical education: a scoping review. Medical Science Educator, pages 1-8.

De Medio, C., Limongelli, C., Marani, A., and Taibi, D. (2019). Retrieval of educational resources from the web: A comparison between google and online educational repositories. In International Conference on Web-Based Learning, pages 28-38. Springer.

Felder, R. M., Silverman, L. K., et al. (1988). Learning and teaching styles in engineering education. Engineering education, 78(7):674-681.

IEEE LTSC (2001). IEEE 1484.12.1 - Draft Standard for Learning Object Metadata. Learning Technology Standards Committee of the IEEE.

Júnior, C. B. and Dorça, F. (2018). Uma abordagem para a criação e recomendação de objetos de aprendizagem usando um algoritmo genético, tecnologias da web semântica e uma ontologia. In Simpósio Brasileiro de Informática na Educação.

Kirschner, P. A. (2017). Stop propagating the learning styles myth. Computers \& Education, 106:166-171.

Ljubojevic, M., Vaskovic, V., Stankovic, S., and Vaskovic, J. (2014). Using supplementary video in multimedia instruction as a teaching tool to increase efficiency of learning and quality of experience. International Review of Research in Open and Distributed Learning, 15(3):275-291.

Lops, P., De Gemmis, M., and Semeraro, G. (2011). Content-based recommender systems: State of the art and trends. In Recommender systems handbook. Springer.

Manouselis, N., Drachsler, H., Vuorikari, R., Hummel, H., and Koper, R. (2011). Recommender systems in technology enhanced learning. In Recommender systems handbook, pages 387-415. Springer.

Pal, S., Pramanik, P. K. D., Majumdar, T., and Choudhury, P. (2019). A semi-automatic metadata extraction model and method for video-based e-learning contents. Education and Information Technologies, 24(6):3243-3268.

Pappas, I. O., Kourouthanassis, P. E., Giannakos, M. N., and Lekakos, G. (2017). The interplay of online shopping motivations and experiential factors on personalized ecommerce: A complexity theory approach. Telematics and Informatics, 34(5).

Selwyn, N. and Gorard, S. (2016). Students' use of wikipedia as an academic resource patterns of use and perceptions of usefulness. The Internet and Higher Education, 28.

Wan, S. and Niu, Z. (2018). An e-learning recommendation approach based on the selforganization of learning resource. Knowledge-Based Systems, 160:71 - 87. 